\title{
Ecolocation: A Sequence Based Technique for RF Localization in Wireless Sensor Networks
}

\author{
Kiran Yedavalli*, Bhaskar Krishnamachari*, Sharmila Ravula ${ }^{\dagger}$, Bhaskar Srinivasan ${ }^{\dagger}$ \\ * Department of Electrical Engineering - Systems \\ University of Southern California, Los Angeles, CA \\ Email: kyedaval@usc.edu,bkrishna@usc.edu \\ ${ }^{\dagger}$ Robert Bosch Research and Technology Center, Palo Alto, CA \\ Email: sharmila.ravula@rtc.bosch.com, bhaskar.srinivasan@rtc.bosch.com
}

\begin{abstract}
In this paper we present a novel sequence-based RF localization algorithm called Ecolocation. Our algorithm determines the location of unknown nodes by examining the ordered sequence of received signal strength (RSS) measurements taken at multiple reference nodes. We employ a constraint-based approach that provides for robust location decoding even in the presence of random RSS fluctuations due to multipath fading and shadowing. Through extensive systematic simulations, and a representative set of real mote experiments, we show that over a wide range of settings Ecolocation performs better than other state of the art approaches in terms of localization accuracy and precision.
\end{abstract}

\section{INTRODUCTION}

Wireless sensor networks (WSN) are severely constrained for energy and cost of deployment and operation. The unique selling point of many WSN systems is that they are inexpensive, autonomous systems capable of working unattended for many years. This can be realized to some extent by multi-tasking the components on sensor motes. Thus, the system radio which is used for inter-mote communication can also be used for localization.

In this paper we present a novel RF based node localization algorithm called Ecolocation that examines the ordered sequence of nearby reference nodes (nodes with known locations) to determine the location of the unknown node (node with unknown location). The key idea of Ecolocation is that the distance-based rank order of reference nodes constitutes a unique signature for different regions in the localization space.

In Ecolocation, we obtain the ordered sequence of reference nodes by ranking them on one-way RSS measurements between them and the unknown node. This measured sequence is then compared with the ideal distance-based sequence for each location to determine how many order-constraints are satisfied. The location which maximizes the number of satisfied constraints is then determined to be the best estimate of the unknown node's location.

Ideally, the ranks of the reference nodes based on RSS readings should be monotonic with their ranks based on true Euclidean distance. Of course, this is not true in the real world because of the presence of multi-path fading and shadowing in the RF channel. Reference nodes farther from the unknown node might measure higher RSS values than those which are closer and this introduces errors in the constraints. However, we show that the inherent insensitivity to absolute RSS amplitudes and the inherent redundancy present in the set of constraints make this approach to localization very robust in practice. Because of the close analogy to controlling errors by redundancy in traditional error control coding, we name our algorithm the "Error COntrolling LOCAlizaTION technique", or Ecolocation for short.

The rest of the paper is organized as follows: Section II describes Ecolocation in detail and presents some illustrative examples. Section III deals with related RF based localization techniques which we use for comparison with Ecolocation. In section IV we evaluate Ecolocation and present its comparative study with other localization techniques. Section V discusses the results of real world systems implementation and the conclusion and future work are presented in section VI.

\section{ECOLOCATION}

In this section we describe Ecolocation and illustrate it for the ideal and real world scenarios through examples.

The localization process is initiated by the unknown node by broadcasting a localization packet. The reference nodes collect RSS measurements of this packet and forward them to a single point ${ }^{1}$ where the location estimate is computed as follows:

1) Determine the ordered sequence of reference nodes by ranking them on the collected RSS measurements.

2) For each possible location grid-point in the location space determine the relative ordering of reference nodes and compare it with the RSS ordering previously obtained, to determine how many of the ordering constraints are satisfied.

3) Pick the location that maximizes the number of satisfied constraints. If there is more than one such location, take their centroid.

\section{A. Ideal versus Real World Scenarios}

Radio frequency (RF) based localization techniques are inherently dependent on the RF channel whose multi-path fading and shadowing effects have a fundamental bearing on the accuracy of location estimate. Nevertheless, it helps to study the localization technique in isolation of these effects. We introduce Ecolocation for the ideal scenario of zero multi-path fading and shadowing effects and latter explain why it provides robust and accurate location estimate even in the presence of these effects.

1) Ideal Scenario: In the absence of multi-path fading and shadowing, RSS measurements between the reference nodes and the unknown node accurately represent the distances between them. If the reference nodes are ranked as a sequence in decreasing order of these RSS values then this order represents the increasing order of their separation from the unknown node. For a reference node ranked at position $i$ in the ordered sequence,

$$
R_{i}>R_{j} \Rightarrow d_{i}<d_{j}, \forall i<j
$$

where, $R_{i}$ and $d_{i}$ are the RSS measurement and distance of the $i^{t h}$ ranked reference node from the unknown node, respectively.

${ }^{1}$ This could be either a cluster-head or the unknown node itself, depending on the application and computational capabilities of nodes. 
The above relationship between two reference nodes is a constraint on the location of the unknown node and is dependent on it. An $i^{\text {th }}$ ranked reference node forms $(i-1)$ constraints with lesser ranked ones and for a total of $\alpha$ reference nodes there are $\left(\frac{\alpha(\alpha-1)}{2}\right)$ constraints on the unknown node.

For fixed reference node locations, the sequence order and the constraints are completely determined by the unknown node location. Figure 1 illustrates this idea for a simple case of five reference nodes and one unknown node.

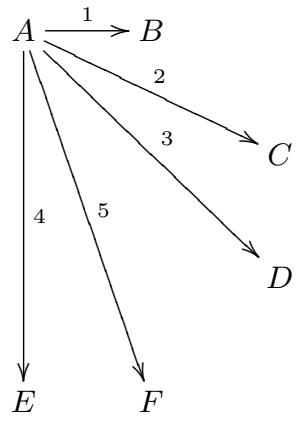

(a)

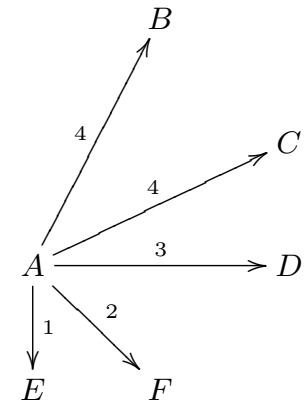

(b)
Fig. 1. The order of reference nodes $(B, C, D, E, F)$ depends on the location of the unknown node $(A)$.

Table I shows the constraints on the unknown node for the example in 1 (a).

\begin{tabular}{|c|c|c|c|c|}
\hline $\mathrm{B}: 1$ & $\mathrm{C}: 2$ & $\mathrm{D}: 3$ & $\mathrm{E}: 4$ & $\mathrm{~F}: 5$ \\
\hline$R_{1}$ & $R_{2}<R_{1}$ & $R_{3}<R_{1}$ & $R_{4}<R_{1}$ & $R_{5}<R_{1}$ \\
& & $R_{3}<R_{2}$ & $R_{4}<R_{2}$ & $R_{5}<R_{2}$ \\
& & & $R_{4}<R_{3}$ & $R_{5}<R_{3}$ \\
& & & & $R_{5}<R_{4}$ \\
\hline
\end{tabular}

TABLE I

CONSTRAINTS ON THE UNKNOWN NODE FOR THE EXAMPLE IN FIGURE 1(A).

Each location grid-point ${ }^{2}$ in the location space has its own set of constraints based on its Euclidean distances to the reference nodes. The unknown node location estimate can be obtained by comparing the constraints obtained from RSS measurements to the constraint sets of each location grid-point and picking the location which satisfies the maximum number of constraints. If there are more than one such locations then their centroid is the location estimate.

2) Real World Scenario: In contrast to the ideal scenario, the real world is characterized by the presence of multi-path fading and shadowing in the RF channel. Ideally, reference nodes that are far from the unknown node should measure lower RSS values than reference nodes that are nearer, but due to multi-path effects this is not true in the real world.

Figure 2 shows the experimental RSS measurements at five MICA 2 receivers placed at different distances from a MICA 2 transmitter. It shows that the receiver at 5.69 meters measured a higher RSS value than the receiver at 5.37 meters. Evidently, RSS measurements do not represent distances accurately in the real world.

Therefore, if the reference nodes are ranked on their respective RSS measurements, the constraints on the unknown node location

${ }^{2}$ Location space scanning can be made more efficient by using greedy search/multiresolution algorithms instead of exhaustively looking at all locations, but we do not discuss this optimization in this paper as it doesn't affect localization performance.

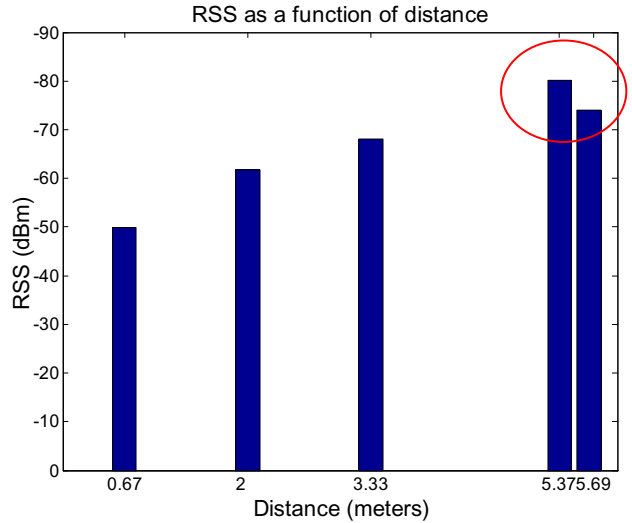

Fig. 2. Real world experimental results: Reference nodes far from the unknown node may measure higher RSS values than closer reference nodes. Note that $\mathrm{y}$-axis is reverse ordered.

formed by these ranks will be erroneous. For example, if the ranks of fourth and fifth ranked reference nodes are interchanged due to multi-path effects in the RF channel, as in the experiment of figure 2, for the example in figure 1(a), then the new constraints are as shown in table II. As it can be seen, $10 \%$ of the constraints are erroneous in this case.

\begin{tabular}{|c|c|c|c|c|}
\hline $\mathrm{B}: 1$ & $\mathrm{C}: 2$ & $\mathrm{D}: 3$ & $\mathrm{E}: 5$ & $\mathrm{~F}: 4$ \\
\hline$R_{1}$ & $R_{2}<R_{1}$ & $R_{3}<R_{1}$ & $R_{5}<R_{1}$ & $R_{4}<R_{1}$ \\
& & $R_{3}<R_{2}$ & $R_{5}<R_{2}$ & $R_{4}<R_{2}$ \\
& & & $R_{5}<R_{3}$ & $R_{4}<R_{3}$ \\
& & & & $R_{4}<R_{5}$ \\
\hline
\end{tabular}

TABLE II

CONSTRAINTS FOR THE EXAMPLE OF TABLE I WHEN THE RANKS OF FOURTH AND FIFTH RANKED REFERENCE NODES ARE INTERCHANGED DUE OF MULTI-PATH EFFECTS.

The percentage of erroneous constraints depends on the RF channel condition, the topology of the reference nodes and the number of reference nodes. The unknown node location estimate accuracy in turn depends on the percentage of erroneous constraints. This is illustrated through a few examples.

Figure 3 shows a sample layout of nine reference nodes placed in a grid and a single unknown node. Figure 3(a) plots the location estimate for the ideal case when there are no erroneous constraints on the unknown node. Figures 3(b), 3(c) and 3(d) show the location estimates for varying percentages of erroneous constraints. It is evident that location estimate error increases with increasing percentage of erroneous constraints.

These examples suggest that Ecolocation is robust to multi-path effects of the RF channel up to some level. The inherent redundancy in the constraint set ensures that the non-erroneous constraints help in estimating the unknown node location accurately. Also, the constraint construction inherently holds true for random variations in RSS measurements up to a tolerance level of $\left(\left|R_{i}-R_{j}\right|\right)$.

For ease of implementation, the constraint set is represented by a constraint matrix $M_{\alpha \times \alpha}$, where

$$
M_{\alpha \times \alpha}(i, j)=\begin{array}{ll}
1 & \text { if } R_{i}<R_{j} \\
0 & \text { if } R_{i}=R_{j} \\
-1 & \text { if } R_{i}>R_{j}
\end{array}
$$




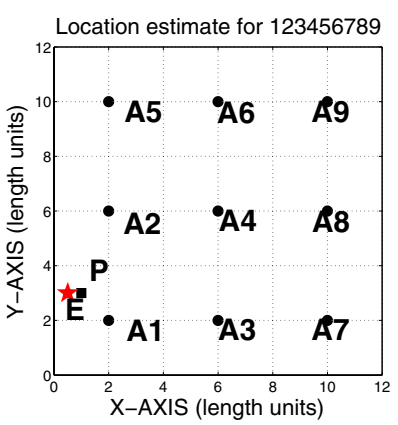

(a)

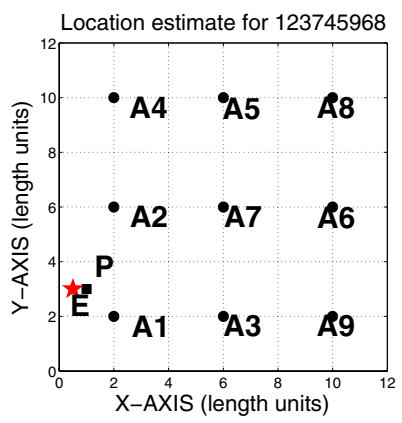

(b)

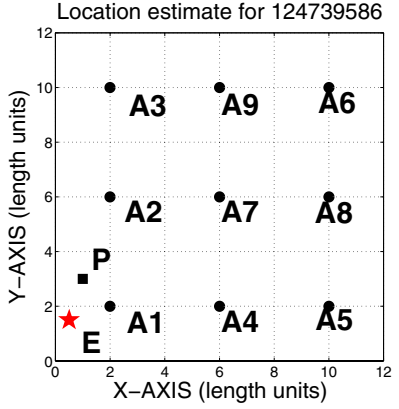

(c)

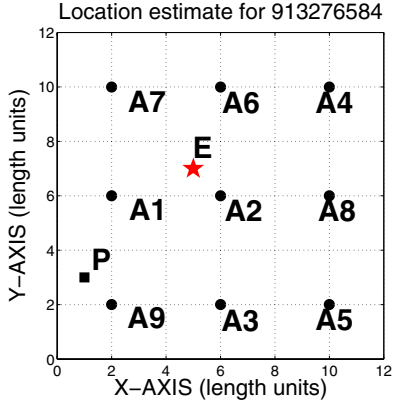

(d)

Fig. 3. Ecolocation location estimate (E) for the unknown node $(\mathrm{P})$ at $(1,3)$ for a grid layout of 9 reference nodes $(\mathrm{A})$. The reference nodes are numbered according to their rank in the ordered sequence. (a) Sequence: 123456789 (no erroneous constraints) [Estimate: (0.5, 3)] (b) Sequence: 123745968 (13.9\% erroneous constraints) [Estimate: $(0.5,3)$ ] (c) Sequence: 124739586 (22.2\% erroneous constraints) [Estimate: $(0.5,1.5)$ ] (d) Sequence: 913276584 (47.2\% erroneous constraints) [Estimate: $(5,7)]$.

It is easy to see that $M_{\alpha \times \alpha}$ is a symmetric matrix and each element of the matrix represents a constraint in the constraint set. The pseudo code for the Ecolocation algorithm is presented below.

ECOLOCATION Input: The number of reference nodes within the range of the unknown node $(\alpha)$, their locations $\left(p_{i x}, p_{i y}\right)(i=$ $1 \ldots \alpha)$, the RSS values of RF signals from the unknown node at each one of them $R_{i}(i=1 \ldots \alpha)$, the localization area size $(\lambda \times \lambda$ sq. length units), and the area scanning resolution $(\gamma)$. Output: The location estimate of the unknown node. The reference nodes are sorted into an ordered sequence based on $R_{i}^{\prime} s$ and a constraint matrix $M_{\alpha \times \alpha}$ is derived from this sequence.

- Calculate the number of matched constraints at each grid point $(i, j)$ and identify the maximum number of constraints matched over all the grid points.

$0 \quad \max$ Constr Match $\leftarrow 0$;

1 for each grid point $(i, j)$ in the localization area

for each reference node $k(\rightarrow 1 \ldots \alpha)$

$d_{k}^{i j} \leftarrow\left(\left(p_{k x}-i\right)^{2}+\left(p_{k y}-j\right)^{2}\right)^{\frac{1}{2}}$

generate constraint matrix $C_{\alpha \times \alpha}^{i j}$ based on $d^{i j}$.

for each element $(m, n)(n>m)$ in $C_{\alpha \times \alpha}^{i j}$

if $C_{\alpha \times \alpha}^{i j}(m, n)=M_{\alpha \times \alpha}(m, n)$ constrMatch $^{i j} \leftarrow$ constrMatch ${ }^{i j}+1$; else

constr Match $^{i j} \leftarrow$ constr Match ${ }^{i j}-1$;

if constrMatch ${ }^{i j}>\max$ ConstrMatch

maxConstrMatch $\leftarrow$ constrMatch ${ }^{i j}$;

- Search for grid points where the maximum number of constraints are matched and return the centroid of those grid points as the location estimate.

$12(x, y) \leftarrow(0,0)$;

13 count $\leftarrow 0$;

14 for each grid point $(i, j)$

15 if constrMatch $^{i j}=$ maxConstrMatch

$16 \quad(x, y) \leftarrow(x+i, y+j)$;

$17 \quad$ count $\leftarrow$ count +1 ;

18 return $\left(\frac{x}{\text { count }}, \frac{y}{\text { count }}\right)>$ Location Estimate.

Complexity Analysis: We should say first of all that this implementation of Ecolocation is meant only to be functional, it is not at all optimized for space or time complexity. Still, the following analysis provides an upper bound on the computational costs for implementing this technique. The initial sorting of reference nodes based on $R_{i}^{\prime} s$ costs $\Theta(\alpha \log (\alpha))$ time and $O(\alpha)$ space respectively. The corresponding constraint matrix generation costs $O\left(\alpha^{2}\right)$ time and $O\left(\alpha^{2}\right)$ space respectively. Calculating the number of constraints matched at each grid point and identifying the maximum number of constraints matched over all grid points (lines 1-11) costs $O\left(\frac{\lambda^{2} \alpha^{2}}{\gamma^{2}}\right)$ time and $O\left(\frac{\lambda^{2}}{\gamma^{2}}+\alpha^{2}\right)$ space respectively. Searching for grid points where maximum number of constraints are matched (lines 12 17) costs $O\left(\frac{\lambda^{2}}{\gamma^{2}}\right)$ time and $O(1)$ extra space. Finally calculating the centroid of those grid points (line 18) costs $O(1)$ time and space. In total, the time and space complexities of Ecolocation are at most $O\left(\frac{\lambda^{2} \alpha^{2}}{\gamma^{2}}\right)$ and $O\left(\frac{\lambda^{2}}{\gamma^{2}}+\alpha^{2}\right)$ respectively ${ }^{3}$. Prior to presenting a complete performance evaluation of Ecolocation, we discuss related localization techniques proposed by others.

\section{RELATED WORK}

Over the past few years many solutions have been proposed for RF-only localization in wireless ad-hoc and sensor networks which can be broadly classified into two main categories - range based and range free. Range based techniques estimate distances (range) from RSS measurements between the unknown node and the reference nodes and use them to triangulate the location of the unknown node [4], [7], [8], [9], [10], [11], [13], [14], [15], [16]. On the other hand range free techniques estimate the location of the unknown node without determining the range [5], [18].

To compare with Ecolocation, we selected four localization techniques - proximity localization, centroid [18], approximate point in triangle [5] and maximum likelihood estimation [8] - based on the criterion that they should use RSS of RF signals to calculate the location estimate over a single hop.

Proximity localization: It is a simple localization scheme in which the location of the closest reference node, based on RSS measurements, is the unknown node location estimate. It can be considered as an extreme special case of Ecolocation where only the first ranking reference node is considered.

Centroid : In [18] the authors propose a range free, proximity based solution for localization where the location estimate is the centroid of all the reference nodes which are in the proximity of the unknown node. In [17] the authors suggest an enhancement to this technique by adaptively placing reference nodes to minimize location error. We

\footnotetext{
${ }^{3}$ We believe the complexities can be significantly reduced by using greedy descent or more efficient scanning versions of the algorithm; this is the subject of ongoing work.
} 
do not consider this enhancement as this requires extra information gathering and processing.

Approximate point in triangle: $\mathrm{T}$. He et al in [5] propose a range free localization technique called approximate point in triangle (APIT) in which the RSS value at the unknown node is compared with RSS values at its neighbors and based on this comparison a decision is made whether the unknown node location is inside various triangles formed by the reference nodes. This comparison test is done for all the locations in the location space and for all the triangles that can be formed by the reference nodes. The location estimate is the centroid of the locations which are in a maximum number of triangles. The accuracy of the location estimate also depends on the non reference node neighbor density of the unknown node.

Maximum Likelihood Estimation: Out of the many maximum likelihood location estimation (MLE) techniques proposed, [4], [14], [8] etc., we consider a simple, representative MLE technique proposed in [8]. In this, the authors calculate the location which maximizes a likelihood function, which is based on the distance estimate and its standard deviation, using the gradient climbing method. All RF based MLE methods need good ranging techniques that use radio frequencies to estimate distances. This either requires expensive ranging equipment and/or time consuming pre-configuration surveys of the location space.

The readers should refer to [1] for a detailed description, including the pseudo code and scalability analysis, for the above four localization techniques.

In [3] the authors present a comparative study of many RSS based localization techniques using commodity 802.11 cards. According to the authors none of the localization techniques have a significant advantage over others over a range of environments. We conjecture that this could be an artifact of fixing the number of nodes and the node density. Our work differs from this in evaluating the performance of five different RSS based localization techniques over different node deployments in different RF channel conditions.

\section{EVALUATION}

In this section we present a complete performance evaluation of Ecolocation using simulations.

\section{A. Simulation Model}

The most widely used simulation model to generate RSS samples as a function of distance in RF channels is the log-normal shadowing model [19]:

$$
R S S(d)=P_{T}-P L\left(d_{0}\right)-10 \eta \log _{10} \frac{d}{d_{0}}+X_{\sigma}
$$

where, $P_{T}$ is the transmit power and $P L\left(d_{0}\right)$ is path loss for a reference distance of $d_{0} . \eta$ is the path loss exponent and the random variation in RSS is expressed as a gaussian random variable of zero mean and $\sigma^{2}$ variance, $X_{\sigma}=N\left(0, \sigma^{2}\right)$. All powers are in $d B m$ and all distances are in meters. In this model we do not provision separately for any obstructions like walls. If obstructions are to be considered an extra constant needs to be subtracted from equation (1) to account for the attenuation in them (the constant depends on the type and number of obstructions).

\section{B. Simulation Parameters}

The location estimate of any RF-based localization technique depends on a fundamental set of parameters which can be broadly categorized into RF channel characteristics and node deployment parameters.
- RF Channel Characteristics: [20], [19]

- Path loss exponent $(\eta)$ : Measures the power attenuation of RF signals relative to distance.

- Standard deviation $(\sigma)$ : Measures the standard deviation in RSS measurements due to log-normal shadowing.

The values of $\eta$ and $\sigma$ change with the frequency of operation and the clutter and disturbance in the environment.

- Node Deployment Parameters:

- Number of reference nodes $(\alpha)$ and unknown nodes $(\rho)$.

- Density of reference nodes $(\beta)$ and unknown nodes. Node density is defined as the number of nodes per square meter.

- Location space size: A square area of $(\lambda \times \lambda)$ sq. meters is considered.

- Resolution or granularity $(\gamma)$ : The unit distance between two grid points in the location space.

- Node distribution in the location space: Random, grid topology or grid-random topology.

The effect of each of the above parameters on any localization technique depends on the actual technique itself. For example, some localization techniques depend more on the number of reference nodes than resolution, while for some other techniques reference node density may be more important than the number of reference nodes.

Table III lists the typical values and ranges for different parameters used in our simulations. Each simulation scenario consists of randomly placing $\alpha$ reference nodes and one unknown node in a square of $(\lambda \times \lambda)$ square meters and generating RSS values between them using equation 1 . A 48 bit arithmetic, linear congruential pseudo random number generator was used and results were averaged over 100 random trials using 10 different random seeds.

\begin{tabular}{|c|c|c|}
\hline Parameter & Typical Value & Typical Range \\
\hline \hline$P_{T}$ & $4 \mathrm{dBm}$ (max.) & NA \\
\hline$P L\left(d_{0}\right)$ & $55 \mathrm{~dB}\left(d_{0}=1 \mathrm{~m}\right)[2]$ & NA \\
\hline$\eta$ & 4 (indoors) & $1-7[20]$ \\
& 4 (outdoors) & \\
\hline$\sigma$ & 7 (indoors) & $2-14[20]$ \\
& 5 (outdoors) & $3-25$ \\
\hline$\alpha$ & 25 & $\{50,25,15,5\}$ \\
\hline$\lambda$ & 15 & $\{0.01,0.04,0.11,1\}$ \\
& 0.11 & \\
\hline$\beta\left(=\frac{\alpha}{\lambda^{2}}\right)$ & $\begin{array}{c}1 \text { ref. node in } \\
9 \text { sq.meters) }\end{array}$ & NA \\
\hline$\gamma$ (for APIT) & 0.1 & NA \\
\hline Node & 8 & Grid-random) \\
Placement & Random & \\
\hline
\end{tabular}

TABLE III

TYPICAL VALUES AND RANGES FOR DIFFERENT SIMULATION PARAMETERS

\section{Simulation Results}

The performance of Ecolocation is measured on two metrics - (i) location error and (ii) location precision - for a wide range of RF channel conditions and node deployment parameters. A comparative study of Ecolocation with the four localization techniques described in section III is also presented.

Location error is defined as the Euclidean distance between the location estimate and the actual location of the unknown node and location precision is defined as the standard deviation in the location error. Location precision is a measure of the robustness of the localization technique as it reveals the variation in its performance 


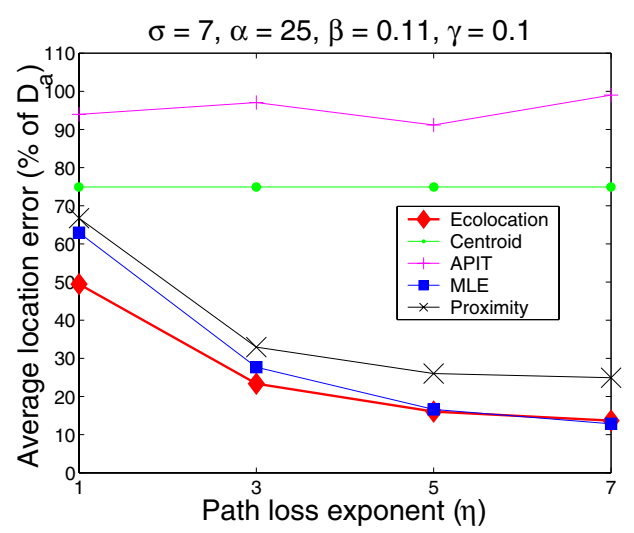

(a)

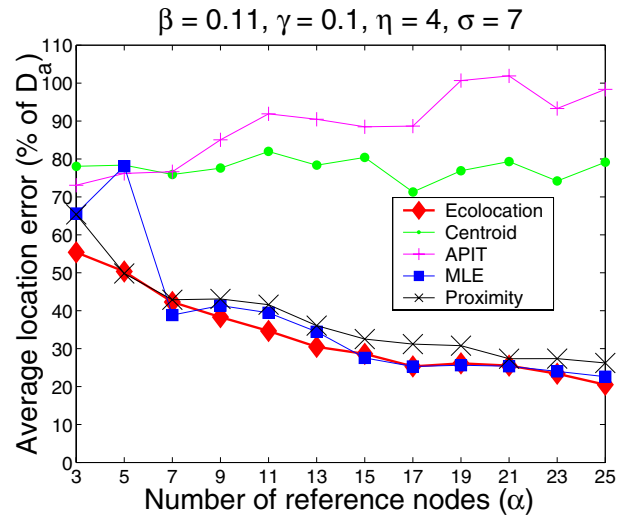

(c)

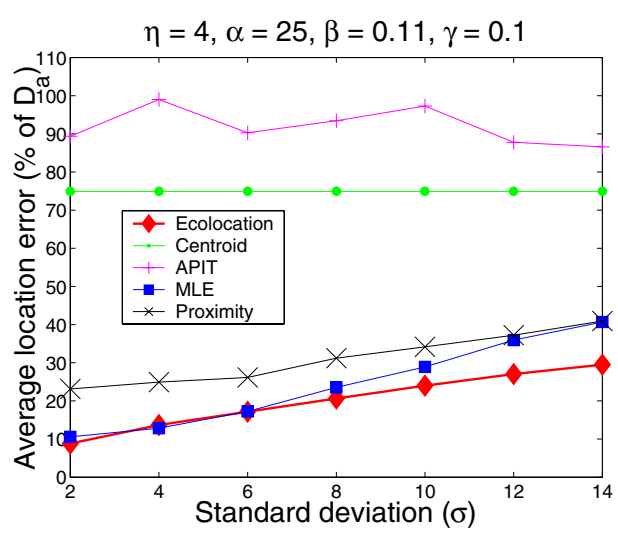

(b)

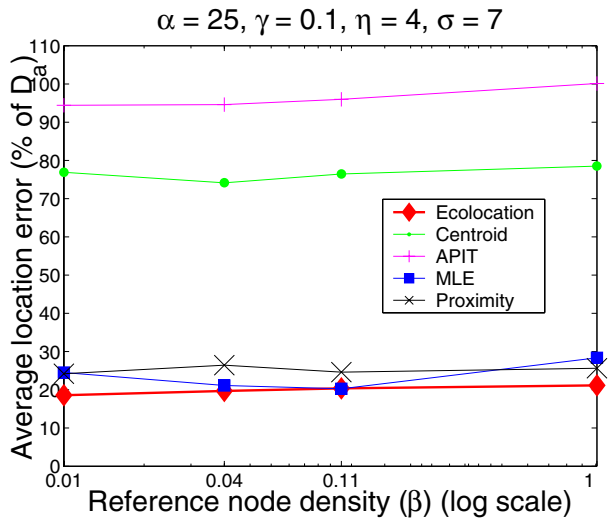

(d)

Fig. 4. Comparison: (a) Average location error as a function of path loss exponent $(\sigma=7, \alpha=25, \beta=0.11, \gamma=0.1)$ (b) Average location error as a function of standard deviation $(\eta=4, \alpha=25, \beta=0.11, \gamma=0.1)$ (c) Average location error as a function of number of reference nodes $(\eta=4, \sigma=7$, $\beta=0.11, \gamma=0.1$ ) (d) Average location error as a function of reference node density $(\eta=4, \sigma=7, \alpha=25, \gamma=0.1)$.

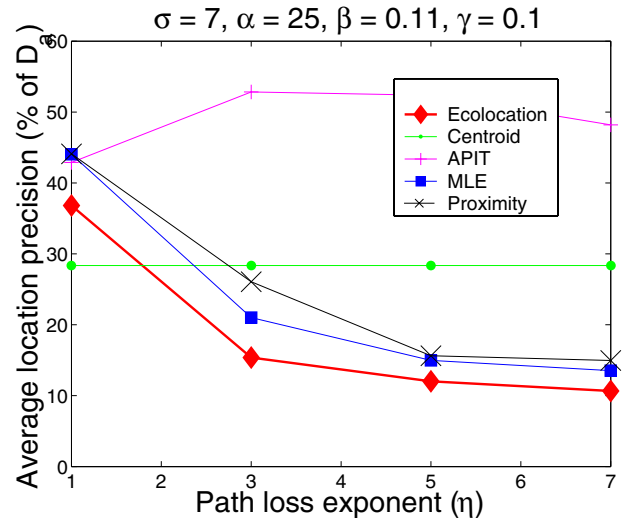

(a)

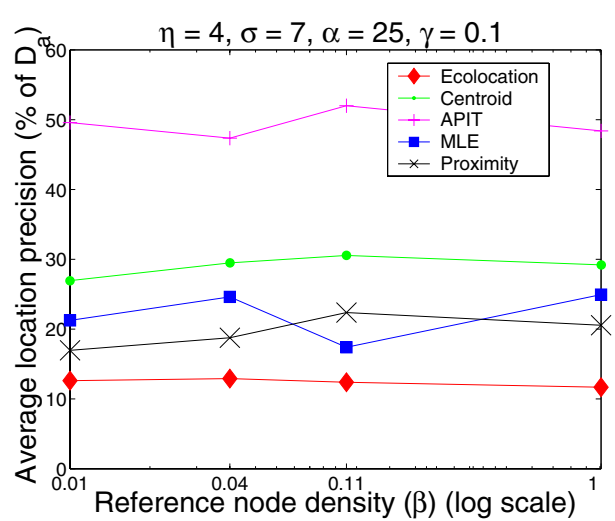

(b)

Fig. 5. Comparison: (a) Average location precision as a function of path loss exponent $(\sigma=7, \alpha=25, \beta=0.11, \gamma=0.1)$ (b) Average location precision as a function of reference node density $(\eta=4, \sigma=7, \alpha=25, \gamma=0.1)$.

over many trials. The two metrics are averaged over 100 random trials and presented as a percentage of the average inter reference node distance $\left(D_{a}\right)$. The distance $D_{a}$ is calculated as the average of the distances between all possible reference node pairs. $\left(D_{a} \approx \frac{\lambda}{2}\right)$.

Figures 4(a) and 4(b) show the average location error for Ecolocation and the four localization techniques as a function of path loss exponent $(\eta)$ and standard deviation of log-normal shadowing $(\sigma)$ respectively. The results suggest that Ecolocation performs better for
RF channels that have higher $\eta$ and lower $\sigma$ values.

Among all five localization techniques Ecolocation provides the least location error over a range of $\eta$ and $\sigma$ values. MLE performs equally well for some values. APIT is the least accurate and Centroid is not influenced by radio channel conditions because all reference nodes fall in the radio range of the unknown node.

Figures 4(c) and 4(d) compare the average location error for all five localization techniques as a function of the number of reference nodes 
$(\alpha)$ and the reference node densities $(\beta)$ respectively. As the results show, Ecolocation performance improves with increasing reference nodes numbers and density. It should be noted that the average inter reference node distance $\left(D_{a}\right)$ changes with reference node density. Although, figure 4(d) shows that the error due to Ecolocation is constant over all densities, it is constant as a percentage of $D_{a}$ implying that the absolute location error increases with reducing density of reference nodes.

Ecolocation provides the most accurate location estimate compared to other techniques over a range of reference node numbers and their densities. Proximity localization performs surprisingly well compared to APIT and Centroid. As expected, the performance of Ecolocation, MLE and Proximity localization improves with number of reference nodes, whereas, it degrades for Centroid and APIT. The results showed that the average location error due to APIT does not decrease with increasing number of reference nodes as suggested in the original paper [5]. We believe this is because the results presented in [5] are based on a simpler model where RSS values decrease monotonically with distance.

Figures 5(a) and 5(b) compare the average location precision for Ecolocation and the four localization techniques as a function of path loss exponent $(\eta)$ and reference node density $(\beta)$ respectively. These figures show that Ecolocation is more robust than other localization techniques over a range of RF channel characteristics and node deployment parameters.

Figure 6 plots the average location error due to Ecolocation for different reference node distributions and it shows that grid placement of reference nodes provides the best location estimates and random placement provides the worst. This is expected because, reference nodes are spread more widely in grid placement than in random placement resulting in a more robust constraint set.

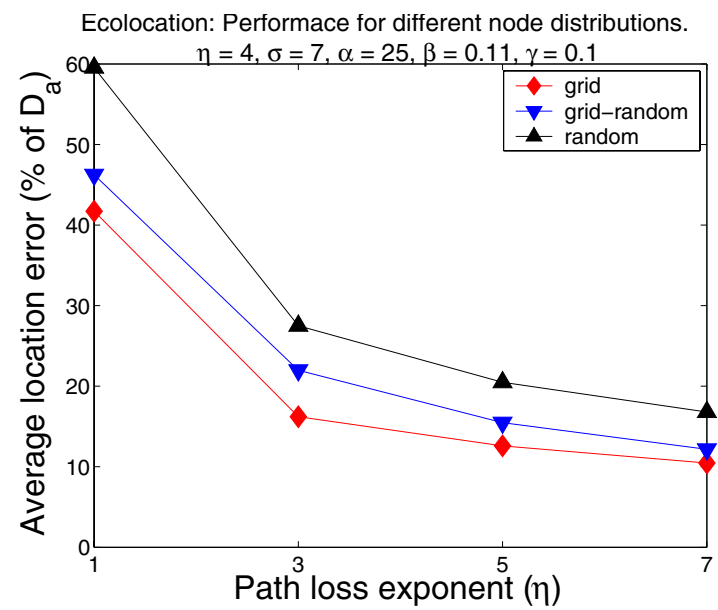

Fig. 6. Average location error as a function path loss exponent for different reference node distributions $(\eta=4, \sigma=7, \alpha=25, \beta=0.11, \gamma=0.1)$.

Simulation results suggest that Ecolocation and MLE perform equally well for some radio channel and node deployment parameters even though they follow entirely different approaches. But in the real world, MLE may not perform so well as it needs good $\eta$ and $\sigma$ estimates for the deployment environment and it is very difficult to obtain accurate estimates for these parameters in the presence of obstructions like walls and furniture.

\section{Systems IMPLEMENTATION AND EVALUATION}

The performance of Ecolocation in real systems is studied through two experiments, representing different RF channel and node deployment parameters, conducted using MICA 2 motes. The first experiment was conducted in a parking lot which represents a relatively obstruction free RF channel and the second experiment was conducted in an office building with many rooms and furniture that represents a typical indoor environment.

The locations of the unknown nodes were estimated using three different localization techniques - Ecolocation, MLE and Proximity. APIT and Centroid methods were not considered as the simulation studies showed that their performance is significantly worse than the three chosen localization techniques.

\section{A. Experiment I: Parking lot}

The RF channel in an outdoor parking lot represents a class of relatively obstruction free channels. Eleven MICA 2 motes were placed randomly on the ground as shown in figure 7. All motes were in line of sight of each other and all of them were programmed to broadcast twenty packets without interfering with each other. The motes recorded the RSS values of the received packets and stored them in their EEPROMs. The averages of these RSS values were calculated off-line and used for location estimation.

The locations of all the motes were estimated and compared with their true locations. Since all motes were in radio range of each other each mote had ten reference nodes. For the MLE method, to estimate the distances between the motes, the RSS model described by equation 1 in section IV was used as there were no obstructions between motes in this experiment. The performance of the MLE technique heavily depends on accurate $\eta$ and $\sigma$ estimates for the area in which the experiment was conducted. For this experiment we used values estimated for an identical setup, from the literature, as listed in table III.

Figure 7 compares the true mote locations with Ecolocation location estimates for all the motes. Figure 8 plots the error at each mote location as a percentage of the average inter-reference nodes distance $\left(D_{a}\right)$, due to all the three techniques. Evidently, Ecolocation performs better than MLE and Proximity in most of the cases.

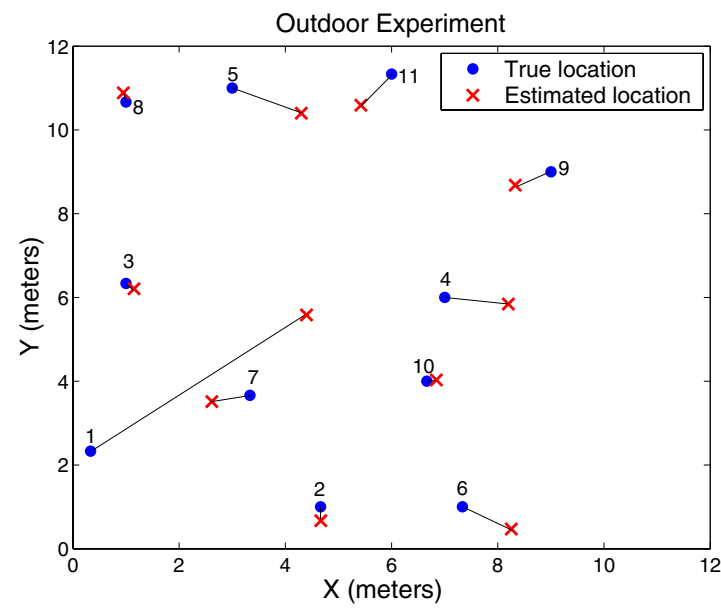

Fig. 7. Outdoor experiment: Comparison between true locations and Ecolocation location estimates. 11 MICA 2 motes, placed randomly in a 144 sq.meters area, were used as reference nodes as well as unknown nodes. Consequently, each unknown node had 10 reference nodes. 


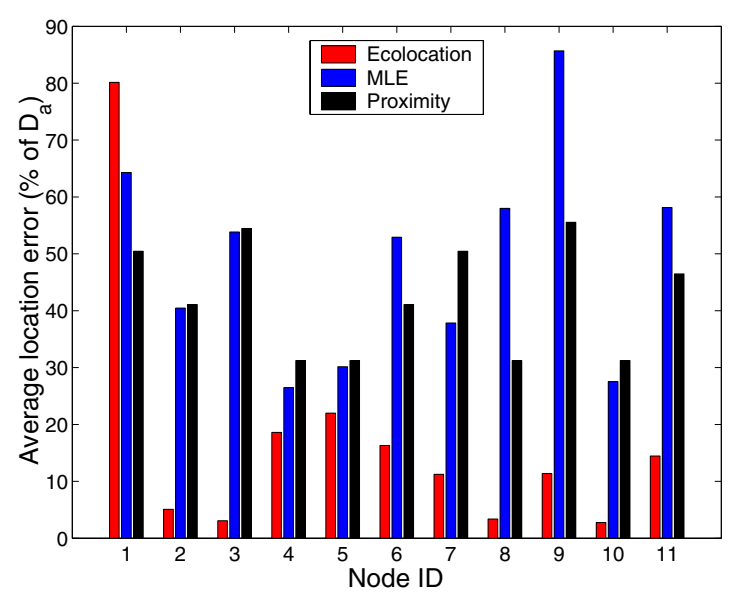

Fig. 8. Location error due to Ecolocation, MLE and Proximity for the outdoor experiment.

\section{B. Experiment II: Office building}

Office buildings with features such as rooms, corridors, furniture and other obstructions represent a distinct class of RF channels. Twelve MICA 2 motes (reference nodes) were placed on the ground randomly in a corner of the Electrical Engineering building at USC spanning different rooms and corridors. Figure 9 shows a schematic of the experimental setup. In this experiment, an unknown node was placed at five different locations and these locations were estimated using all the twelve motes as reference nodes. As in the outdoor experiment, the unknown node was programmed to broadcast twenty packets from each location and the reference nodes recorded the RSS values of these packets in their respective EEPROMs. The average of these RSS readings was used off-line for location estimation.

Unlike in the outdoor experiment not all motes were in line of sight of each other even though they were in each other's radio range. A subset of the motes had obstructions in between them in the form of office walls. Therefore, for the MLE method of localization, the RSS model of equation 1 in section IV was modified to incorporate the power attenuation due to these obstructions. According to [19], dry office walls attenuate RF power by about $4 d B$. This value was multiplied by the number of walls between the unknown node and reference nodes and added to the received signal strength at the reference nodes in order to compensate for the power loss. It should be noted that in real world scenarios it is very difficult to estimate the number of obstructions between two wireless devices. As in the outdoor experiment, the values of $\eta$ and $\sigma$ were obtained from the literature as listed in table III.

Figure 9 compares the Ecolocation location estimates of the five unknown node locations with their true locations. It can be seen that the path of the location estimates closely follows the true path of the unknown node. Figure 10 plots the location estimate error due to Ecolocation, MLE and Proximity techniques for each unknown node location. It can be observed that the error due to Ecolocation is lower than the errors due to MLE and proximity in three out of five cases.

\section{Discussion}

Experimental results show that localization techniques are more accurate for relatively clutter free RF channel environments (outdoors with line of sight) than RF channels with many obstructions (indoor environment). Also, the performance of MLE in real world scenarios is worse than in simulations, as was suggested in section IV. This

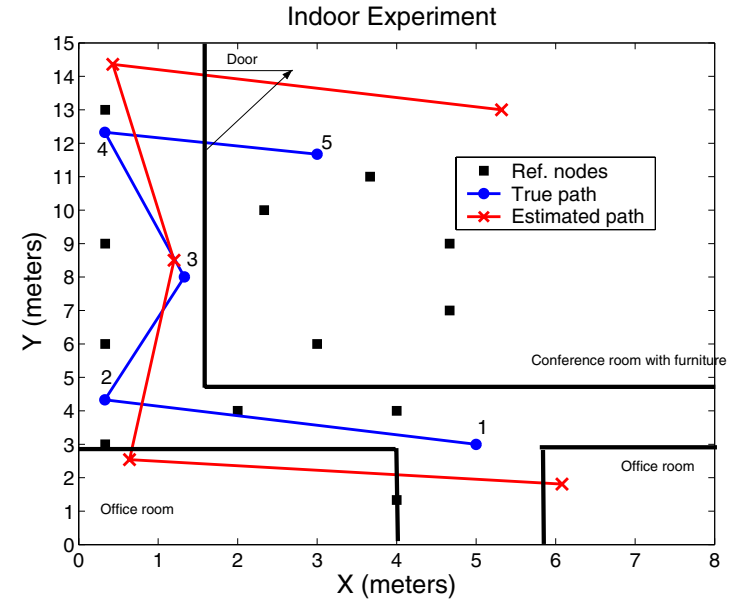

Fig. 9. Indoor experiment: Comparison between true path and Ecolocation estimated path. 12 MICA 2 motes, placed randomly in a 120 sq.meters area, were used as reference nodes. The location of the unknown node was estimated for 5 different locations using the 12 reference nodes.

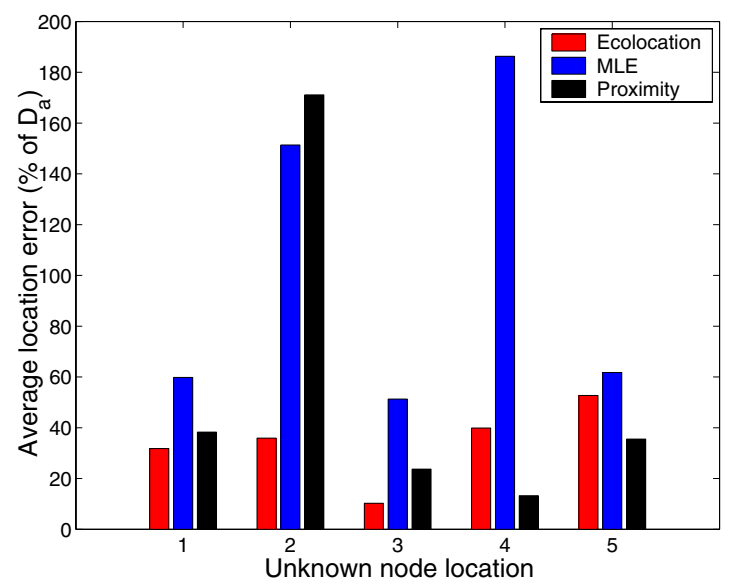

Fig. 10. Location error due to Ecolocation, MLE and Proximity for the indoor experiment.

is mainly because the radio propagation model of equation 1 is an approximate model and the location estimate accuracy for the MLE technique depends heavily on the accuracy of $\eta$ and $\sigma$ values. The RSS measurements in the experiments depend on antenna orientations, antenna height and transmitter/receiver non-determinism. For simulations, these issues can be captured within the log-normal random term in equation 1 of section IV.

Simulation results in section IV show that the performance of Ecolocation is better than that of Proximity by about $10 \%$ on an average. But from figures 8 and 10 it is clear that for individual cases the performance of Ecolocation could be much better than that of Proximity.

From figures 8 and 10 it is clear that a single localization technique may not provide the best accuracy for all unknown node locations even though for a majority of the cases Ecolocation performs better than other techniques. This suggests that a hybrid localization technique which switches between different localization techniques, depending on the RF channel characteristics and node deployment parameters, may provide better accuracy than any single localization technique. 


\section{CONCLusion AND Future Work}

In this paper we presented a novel RF based localization technique called Ecolocation which makes use of the inherent redundancy present in the ordering of reference nodes with respect to the unknown node to provide good location accuracy. The comparative study of Ecolocation with four other RF based localization techniques, through simulations and real world systems implementation, shows that Ecolocation performs better than other localization techniques over a range of RF channel conditions and node deployment strategies.

In the future we would like to explore area scanning algorithms such as greedy search and multi-resolution search for making Ecolocation more efficient. All localization techniques extract costs from the system over which they operate. We plan to study the effect of Ecolocation as well as other localization techniques over a variety of realistic system designs and protocols. We would also like to explore the different quantities which can be used for switching purposes in a hybrid localization technique.

\section{REFERENCES}

[1] Kiran Yedavalli, Bhaskar Krishnamachari, Sharmila Ravula, Bhaskar Srinivasan, Ecolocation: A Sequence Based Technique for RF Localization in Wireless Sensor Networks, USC CENG Technical Report number 2004-16.

[2] Marco Zuniga, Bhaskar Krishnamachari, Analyzing the Transitional Region in Low Power Wireless Links, First IEEE International Conference on Sensor and Ad hoc Communications and Networks (SECON), Santa Clara, CA, October 2004.

[3] Eiman Elnahrawy, Xiaoyan Li, Richard P. Martin, The Limits of Localization Using Signal Strength: A Comparative Study, First IEEE International Conference on Sensor and Ad hoc Communications and Networks (SECON), Santa Clara, CA, October 2004.

[4] N. Patwari and A.O. Hero III, Using Proximity and Quantized RSS for Sensor Localization in Wireless Networks. WSNA'03. San Diego, CA, September 2003.

[5] T. He, C. Huang, B.M. Blum, J.A. Stankovic and T. Abdelzaher, RangeFree Localization Schemes for Large Scale Sensor Networks, MobiCom'03, San Diego, Ca, September 2003.
[6] Y. Shang, W. Rumi and Y. Zhang, Localization from Mere Connectivity, MobiHoc'03, Annapolis, Maryland, June 2003.

[7] D. Niculescu and B. Nath, Localized positioning in ad hoc networks. Proceedings of the First IEEE International Workshop on Sensor Network Protocols and Applications, 11 May 2003.

[8] Kiran Yedavalli, Location Determination Using IEEE 802.11b, MS Thesis, University of Colorado, Boulder, December 2002.

[9] A. Savvides, H. Park and M.B. Srivastava, The Bits and Flops of the Nhop Multilateration Primitive For Node Localization Problems. WSNA'02, Atlanta, Georgia, September 2002.

[10] A. Nasipuri and K. Li, A Directionality based Location Discovery Scheme for Wireless Sensor Networks, WSNA'02, Atlanta, Georgia, September 2002.

[11] P. Bergamo and G. Mazzini, Localization in sensor networks with fading and mobility. The 13th IEEE International Symposium on Personal, Indoor and Mobile Radio Communications (PIMRC'02), Volume: 2, September 2002

[12] D. Niculescu and D. Nath, Ad-Hoc Positioning Systems (APS), In Proceedings of IEEE GLOBECOM '01, November 2001.

[13] J. Albowicz, A. Chen and L. Zhang, Recursive position estimation in sensor networks, Ninth International Conference on Network Protocols, November 2001.

[14] A. Savvides, C.C. Han and M.B. Srivastava, Dynamic Fine Grained Localization in Ad-Hoc Sensor Networks, Proceedings of the Fifth International Conference on Mobile Computing and Networking, Mobicom 2001, pp. 166-179, Rome, Italy, July 2001.

[15] C. Savarese, J.M. Rabaey and J. Beutel. Locationing in Distributed AdHoc Wireless Sensor Networks, ICASSP, May 2001.

[16] L. Doherty, K.S.J. Pister and L.E. Ghaoui, Convex Position Estimation in Wireless Sensor Networks, INFOCOM'01, Anchorage, AK, April 2001.

[17] N. Bulusu, J. Heidemann, and D. Estrin, Density Adaptive Algorithms for Beacon Placement in Wireless Sensor Networks, In IEEE ICDCS '01, Phoenix, AZ, April 2001.

[18] N. Bulusu, J. Heidemann, and D. Estrin, GPS-less Low-Cost Outdoor Localization for Very Small Devices, IEEE Personal Communications Magazine, October 2000.

[19] Theodore S. Rappaport, Wireless Communications, Principles \& Practice, Prentice Hall, 1999.

[20] H. Hashemi. The indoor radio propagation channel. Proceedings of the IEEE, 81(7): 943-968, July 1993. 